\title{
Heavy quarkonium and nonperturbative corrections
}

\author{
A. Pineda* and J. Soto ${ }^{\dagger}$ \\ Department d'Estructura i Constituents de la Matèria and Institut de Física d'Altes Energies, Universitat de Barcelona, \\ Diagonal, 647, E-08028 Barcelona, Catalonia, Spain
}

(Received 20 February 1996; revised manuscript received 12 June 1996)

\begin{abstract}
We analyze the possible existence of nonperturbative contributions in heavy $\bar{Q} Q$ systems $(\bar{Q}$ and $Q$ need not have the same flavor) which cannot be expressed in terms of local condensates. Starting from QCD, with well-defined approximations and splitting properly the fields into large and small momentum components, we derive an effective Lagrangian where hard gluons (in the nonrelativistic approximation) have been integrated out. The large momentum contributions (which are dominant) are calculated using Coulomb-type states. In addition to the usual condensate corrections, we see the possibility of new nonperturbative contributions. We parametrize them in terms of two low momentum correlators with Coulomb bound state energy insertions $E_{n}$. We realize that the heavy quark effective Lagrangian can be used in these correlators. We calculate the corrections that they give rise to in the decay constant, the bound state energy, and the matrix elements of bilinear currents at zero recoil. We study the cutoff dependence of the new contributions and we see that it matches perfectly with that of the large momentum contributions. We consider two situations in detail, (i) $E_{n} \gg \Lambda_{\mathrm{QCD}}\left(M_{Q} \rightarrow \infty\right)$ and (ii) $E_{n} \ll \Lambda_{\mathrm{QCD}}$, and briefly discuss the expected size of the new contributions in $Y$, $J / \psi$, and $B_{c}^{*}$ systems. [S0556-2821(96)01419-1]
\end{abstract}

PACS number(s): 12.38.Lg, 12.39.Hg, 13.20.Gd

\section{INTRODUCTION}

The study of heavy quark bound state systems remains one of the more promising topics in order to test both perturbative and nonperturbative aspects of QCD, as is clear from the steady activity in the field [1-6]. These systems can be understood in a first approximation as nonrelativistic bound states which occur due to a Coulomb-type interaction predicted by perturbative QCD. In order to improve this basic picture one has to deal on the one hand with perturbative relativistic and radiative corrections, and on the other hand with nonperturbative corrections (power corrections).

In this paper we shall only be concerned with nonperturbative corrections. Usually, the latter have been parametrized using both the multipole expansion and the adiabatic approximation in terms of the gluon condensate $[7,8]$. Corrections to the Coulomb potential due to condensates can also be considered, although these are subleading [3,9]. We have argued before [6] that new nonperturbative contributions could arise which cannot be expressed in terms of local condensates, and hence a convenient parametrization for them is required. This kind of nonperturbative contribution has been discussed in [10] in a different context and, in fact, the various Isgur-Wise functions extensively used in the heavy quark effective theory (HQET) may be regarded as such [11].

Let us recall the main idea behind the possibility of new nonperturbative contributions in heavy quarkonium. ${ }^{1}$ When the relative three-momentum in the bound state is large enough, the dominant interaction must be the perturbative

\footnotetext{
*Electronic address: pineda@ecm.ub.es

†Electronic address: Soto@ecm.ub.es

${ }^{1}$ We use "heavy quarkonium', to denote a general heavy quarkantiquark bound state. The quark and the antiquark need not have the same flavor.
}

Coulomb potential, but for small relative three-momentum this need not longer be true. Therefore, heavy quarks in the latter kinematical situation should better be kept as lowenergy degrees of freedom. It turns out that a convenient parametrization of this kinematical region may be given in terms of the HQET for quarks and antiquarks [6,12].

The HQET for quarks and antiquarks enjoys rather peculiar features, which make it quite different from the usual HQET describing either quarks or antiquarks, which has been so popular in the study of $Q \bar{q}$ and $Q q q$ systems in recent years [13] (see [14] for reviews). For instance, it enjoys a symmetry, which is larger than the well-known spin and flavor symmetry, that breaks spontaneously down to the latter, giving rise to quark-antiquark states as Goldstone modes [12]. Its peculiarities concerning radiative corrections have recently been illustrated in [15].

The main aim of this paper is to work out a controlled derivation from QCD of the effective Lagrangian describing the small relative momentum regime of heavy quarks in quarkonium. Whereas the basic ideas above have already been elaborated in [6], a complete and systematic derivation is lacking and, hence, worth being presented. Within this new framework we recalculate the nonperturbative contributions of this region to the energy levels, the decay constant, and the matrix elements of bilinear currents at zero recoil. We find a few corrections to the formulas given in [6]. For all these observables it is enough to work in the center-ofmass (c.m.) frame, which we shall do in most of the paper.

In order to deal with heavy quarkonia systems we keep the relevant degrees of freedom in the QCD Lagrangian. In fact, since virtual heavy quark creation is very much suppressed, we could safely start from nonrelativistic QCD (NRQCD). The derivation of NRQCD from QCD is well understood and a technique to incorporate relativistic corrections to it has also been developed [16]. First of all, we split the gluon field into hard and soft components by a threemomentum cutoff. From the hard gluon fields we only keep 
the zero component and disregard the special components. This is legitimate as far as we are not interested in relativistic corrections. We next integrate out the zero component of the hard gluon field to obtain the Coulomb potential between heavy quark currents. The Coulomb potential has an infrared momentum cutoff since the zero component of the soft gluon field has not been integrated out. At this point we have an effective Lagrangian formally equal to the one used by Voloshin and Leutwyler (VL) [7,8], except for the IR cutoff in the Coulomb potential. After introducing c.m. and relative momenta for the bound states we are interested in, we further split the quark fields into large and small relative threemomentum regimes. ${ }^{2}$ The resulting Lagrangian can then be separated into three pieces: $L^{\mu}$, which contains small relative momentum quark fields only, $L_{\mu}+L_{\mu}^{I}$, which contains large relative momentum quark fields only, and $L_{\mu}^{I \mu}$, which contains both small and large relative momentum quark fields. For $L^{\mu}$ we can approximate the Lagrangian to the HQET Lagrangian, where eventually all its powerful symmetries can be used. No Coulomb term remains in this part of the Lagrangian. For $L_{\mu}+L_{\mu}^{I}$ we obtain again the VL starting point Lagrangian except for two facts: Both the Coulomb potential and the Hilbert space are restricted to threemomenta larger than a certain cutoff. Keeping the cutoff much higher than $\Lambda_{\mathrm{QCD}}$ but much smaller than the inverse Bohr radius we may safely assume that the multipole expansion holds for this part of the Lagrangian. If we further assume that the adiabatic approximation also holds, we may proceed in total analogy to VL. The hypothesis above on the cutoff also allows us to treat $L_{\mu}^{I \mu}$ as a perturbation. The various contributions from this perturbation to the different observables can be eventually expressed as correlators of the HQET.

We would like to stress that our formalism is less restrictive than the one used by VL since neither the adiabatic approximation nor the multipole expansion are assumed to hold in the small relative momentum region of the heavy quark fields. Indeed we may always recover the VL results by putting to zero the cutoff which separates large and small relative momentum.
We organize the paper as follows. In Sec. II we derive the effective action for the small relative momentum fields. In Sec. III we calculate the decay constant, the bound state mass, and the matrix element of any bilinear heavy quark current between quarkonia states at zero recoil. The latter is relevant for the study of semileptonic decays at zero recoil. In Sec. IV we prove the cutoff independence of our results. In Sec. $\mathrm{V}$ we study the low-momentum correlators in two situations: the asymptotic limit $\left(M_{Q} \rightarrow \infty\right) E_{n} \gg \Lambda_{\mathrm{QCD}}$, where, using the operator product expansion (OPE) techniques, we see that no new corrections arise, and (ii) $E_{n} \ll \Lambda_{\mathrm{QCD}}$, where the low-momentum contributions are evaluated using an effective "chiral" Lagrangian which incorporates the relevant symmetries of the HQET for quarks and antiquarks. Working in this way we find new nonperturbative contributions which are parametrized by a single nonperturbative constant. We also give preliminary estimations of their size. Section VI is devoted to the conclusions.

\section{EFFECTIVE ACTION}

In this section we derive the effective Lagrangian for heavy quarks and antiquarks in the small relative momentum regime from QCD.

The QCD Lagrangian reads

$$
\mathcal{L}=-\frac{1}{4} F^{2}+\sum_{a} \bar{Q}_{a}\left(i \not D-m_{a}\right) Q_{a}
$$

where

$$
\begin{gathered}
D_{\mu}=\partial_{\mu}-i g V_{\mu}, \quad V=V^{r} T^{r}, \\
F_{\mu \nu}^{r}=\partial_{\mu} V_{\nu}^{r}-\partial_{\nu} V_{\mu}^{r}+g f^{r s t} V_{\mu}^{s} V_{\nu}^{t} .
\end{gathered}
$$

We split the gluon field $V$ in large $A$ and small $B$ momentum modes $V(x)=A(x)+B(x)$. Next we exactly integrate $A_{0}$ and neglect $A_{i}$. The latter would give rise to relativistic corrections. Consistently, at the same point we perform a Foldy-Wouthuysen transformation and keep terms up to $1 / m$. We obtain

$$
\begin{aligned}
L= & -\frac{1}{4} \int d^{3} x F_{B}^{2}+\sum_{a} \int d^{3} x\left(\bar{Q}_{a}\left(i \gamma^{0} D_{0}^{B}-m_{a}\right) Q_{a}+\bar{Q}_{a} \frac{\vec{D}_{B}^{2}}{2 m_{a}} Q_{a}+\bar{Q}_{a} \frac{g \vec{\Sigma} \cdot \overrightarrow{B_{B}}}{2 m_{a}} Q_{a}\right)+O\left(\frac{1}{m_{a}^{2}}\right) \\
& -\frac{g^{2}}{2} \sum_{a a^{\prime}} \int d^{3} x \int d^{3} y \bar{Q}_{a} \gamma^{0} T^{r} Q_{a}(x)\left(\frac{1}{\vec{D}_{B}^{2}}\right)^{r s}(x, y) \bar{Q}_{a^{\prime}} \gamma^{0} T^{s} Q_{a^{\prime}}(y),
\end{aligned}
$$

which is manifestly gauge invariant. ${ }^{3}$ Although, in principle, we could attempt to carry out an explicitely gauge-invariant calculation, in practice, it is most convenient to choose a slightly modified Schwinger gauge for the small momentum gluons:

$$
\left(\vec{z}-\frac{m_{a} \vec{x}+m_{a^{\prime}} \vec{y}}{m_{a}+m_{a^{\prime}}}\right) \vec{B}(z)=0 .
$$

\footnotetext{
${ }^{2}$ The large and small relative momentum regions were denoted as off- and on-shell regions, respectively, in [6].

${ }^{3}$ Similar approaches can be found in the literature [4].
} 
In this gauge $\vec{B}$ in the kinetic and Coulomb terms gives rise to subdominant contributions when the multipole expansion is carried out, which greatly simplifies the calculation. In particular, recall that the propagator in the Coulomb term always carries large momentum (we have not integrated out the small momentum $V_{0}$ which is kept in $B_{0}$ ). Hence the multipole expansion is always made legitimate in the Coulomb term. This allows us to drop $\vec{B}$ in the Coulomb term straight away. As long as we are interested in quark-antiquark bound states only, we may also safely neglect the four-fermion interaction terms involving only quarks or only antiquarks. We next rearrange the quark-antiquark interaction term in a convenient way in order to describe the bound state dynamics. Finally, the effective Lagrangian reads

$$
\begin{aligned}
L= & -\frac{1}{4} \int d^{3} x F_{B}^{2}+\sum_{a} \int d^{3} x\left(\bar{Q}_{a}\left(i \gamma^{0} D_{0}^{B}-m_{a}\right) Q_{a}+\bar{Q}_{a} \frac{\vec{D}_{B}^{2}}{2 m_{a}} Q_{a}\right)+O\left(\frac{1}{m_{a}^{2}}\right) \\
& -\frac{1}{2} \sum_{a a^{\prime} A} m_{a a^{\prime}}^{3} N_{A}^{2} \sum_{s} \int \frac{d^{3} v}{(2 \pi)^{3}} \int \frac{d^{3} q^{\prime}}{(2 \pi)^{3}} \int \frac{d^{3} q}{(2 \pi)^{3}} V^{A}\left(\vec{q}^{\prime}-\vec{q}\right)\left[\tilde{Q}_{a}\left(-m_{a} \vec{v}+\vec{q}, t\right) T^{A} \bar{\Gamma}_{s} \widetilde{Q}_{a^{\prime}}\left(m_{a^{\prime}} \vec{v}+\vec{q}, t\right)\right] \\
& \times\left[\tilde{\bar{Q}}_{a^{\prime}}\left(m_{a^{\prime}} \vec{v}+\vec{q}, t\right) T^{A} \Gamma_{s} \widetilde{Q}_{a}\left(-m_{a} \vec{v}+\vec{q}^{\prime}, t\right)\right],
\end{aligned}
$$

where $A=0, r$ denotes color ( 0 singlet and $r$ octet, $r=1, \ldots, 8)$, $|\vec{q}-\vec{q}|>\mu, \mu$ being the cutoff which separates small and large momenta, and

$$
\begin{gathered}
m_{a a^{\prime}}=m_{a}+m_{a^{\prime}}, \quad \Gamma_{s}=i \gamma_{5} p_{-}, i \gamma^{i} p_{-}, \quad p_{ \pm}:=\frac{1 \pm \gamma^{0}}{2}, \\
N_{A}=\frac{1}{\sqrt{N_{c}}}, \quad \sqrt{2}, \quad T^{A}=1, T^{r},
\end{gathered}
$$

while the potential reads

$$
V^{0}(\vec{p})=-\frac{C_{F} g^{2}}{\vec{p}^{2}}, \quad V^{r}(\vec{p})=\frac{g^{2}}{2 N_{c} \vec{p}^{2}},
$$

where $C_{F}=\left(N_{c}^{2}-1\right) / 2 N_{c}$ and $|\vec{p}|>\mu$ must be understood as due to the cutoff coming from soft gluons. ${ }^{4}$

Written in this way, we can understand the four-fermion Coulomb interaction term as one which creates a quarkantiquark state with central velocity $\vec{v}$ and relative momentum $\vec{q}$ and annihilates a quark-antiquark state with the same center-of-mass velocity $\vec{v}$ and relative momentum $\vec{q}$. Obviously $\vec{v}$ is a conserved quantity in this nonrelativistic approximation. We consider the spin-breaking term as subleading and we will neglect it in the following. Therefore, spin symmetry for both low and high momenta is implicit in the rest of the paper.

If we stopped at this point we would obtain the standard VL results. However, we would like to go beyond and look for new nonperturbative contributions. We observe that quarks with small relative three-momentum only "feel" the Coulomb interaction of quarks with large relative momentum. This suggests for us to perform a splitting of the physical quark and antiquark fields into small and large relative momenta in the bound state. The physical picture behind is that if the relative three-momentum in the bound state is large enough, we can understand it as a perturbative Coulomb-type bound state. But for small relative threemomentum, that is no longer true. For that momentum regime the quark and antiquark fields should be kept as lowmomentum degrees of freedom. That is, in fact, the main idea of this paper. Therefore, let us write down the currents related to the physical quark-antiquark states in momentum space:

$$
\begin{aligned}
J_{\Gamma}^{A, a^{\prime} a}(x)= & \bar{Q}_{a^{\prime}} T^{A} \Gamma Q_{a}(x) \\
= & m_{a a^{\prime}}^{3} \int \frac{d^{3} v}{(2 \pi)^{3}} e^{i m_{a a^{\prime}} \vec{v} \cdot \vec{x}} \int \frac{d^{3} q}{(2 \pi)^{3}} \\
& \times \tilde{\bar{Q}}_{a^{\prime}}\left(-m_{a^{\prime}} \vec{v}-\vec{q}, t\right) T^{A} \Gamma \widetilde{Q}_{a}\left(m_{a} \vec{v}-\vec{q}, t\right) .
\end{aligned}
$$

The matrix $\Gamma$ should be such that it projects over quarkantiquark states according to our nonrelativistic picture. Notice that the time dependence is kept explicit. Furthermore, we split the relative three-momentum with the same cutoff $\mu$ as above. Thus, Eq. (2.9) reads

$$
\begin{aligned}
J_{\Gamma}^{A, a^{\prime} a}(x)= & J_{l, \Gamma}^{A, a^{\prime} a}(x)+J_{h, \Gamma}^{A, a^{\prime} a}(x) \\
= & m_{a a^{\prime}}^{3} \int \frac{d^{3} v}{(2 \pi)^{3}} e^{i m_{a a^{\prime}} \vec{v} \cdot \vec{x}} \int^{\mu} \frac{d^{3} q}{(2 \pi)^{3}} \tilde{\bar{h}}_{a^{\prime}}^{v}(-\vec{q}, t) T^{A} \Gamma \widetilde{h_{a}^{v}}(-\vec{q}, t) \\
& +m_{a a^{\prime}}^{3} \int \frac{d^{3} v}{(2 \pi)^{3}} e^{i m_{a a^{\prime}} \vec{v} \cdot \vec{x}} \int_{\mu} \frac{d^{3} q}{(2 \pi)^{3}} \tilde{\bar{Q}}_{a^{\prime}}\left(-m_{a^{\prime}} \vec{v}-\vec{q}, t\right) T^{A} \Gamma \widetilde{Q}_{a}\left(m_{a} \vec{v}-\vec{q}, t\right),
\end{aligned}
$$


where $\widetilde{Q}_{a}\left(m_{a} \vec{v}+\vec{k}\right)=: \widetilde{h}_{a}^{v}(\vec{k})$. After that we may divide the Lagrangian into three pieces:

$$
L=L_{\mu}+L^{\mu}+L^{I}
$$

$L_{\mu}$ is the piece of the effective Lagrangian containing large momenta only. It reads

$$
\begin{aligned}
L_{\mu}= & \sum_{a} \int d^{3} x\left(\bar{Q}_{a}\left(i \gamma^{0} \partial_{0}-m_{a}\right) Q_{a}+\bar{Q}_{a} \frac{\vec{\nabla}^{2}}{2 m_{a}} Q_{a}\right)-\frac{1}{2} \sum_{a a^{\prime} A} m_{a a^{\prime}}^{3} N_{A}^{2} \sum_{s} \int \frac{d^{3} v}{(2 \pi)^{3}} \int_{\mu} \frac{d^{3} q^{\prime}}{(2 \pi)^{3}} \int_{\mu} \frac{d^{3} q}{(2 \pi)^{3}} V^{A}\left(\vec{q}^{\prime}-\vec{q}\right) \\
& \times\left[\tilde{\bar{Q}}_{a}\left(-m_{a} \vec{v}+\vec{q}, t\right) T^{A} \bar{\Gamma}_{s} \widetilde{Q}_{a^{\prime}}\left(m_{a^{\prime}} \vec{v}+\vec{q}, t\right)\right]\left[\tilde{Q}_{a^{\prime}}\left(m_{a^{\prime}} \vec{v}+\vec{q}^{\prime}, t\right) T^{A} \Gamma_{s} \widetilde{Q}_{a}\left(-m_{a} \vec{v}+\vec{q}^{\prime}, t\right)\right],
\end{aligned}
$$

where $\left|\vec{q}-\vec{q}^{\prime}\right|>\mu$.

In fact, it is nothing but the standard Coulomb Lagrangian, except for the cutoffs. $L^{\mu}$ is the piece of the effective Lagrangian containing small momenta only. It reads

$$
\mathcal{L}^{\mu}=-\frac{1}{4} F_{B}^{2}+\sum_{a}\left\{\bar{h}_{a}^{v}\left(i \gamma^{0} D_{0}^{B}-m_{a}\right) h_{a}^{v}+\bar{h}_{a}^{v} \frac{\vec{D}_{B}^{2}}{2 m_{a}} h_{a}^{v}\right\} .
$$

Notice that Eq. (2.13) does not have the four-fermion Coulomb term. It contains the whole soft gluon Lagrangian as well as the heavy quark and antiquark fields with small relative three-momentum. All the fields in Eq. (2.13) are in the nonperturbative regime of QCD. Notice that if we drop the term in $1 / m_{a}$ and make $h_{a}^{v} \rightarrow e^{-i \gamma^{0} m_{a} x_{0}} h_{a}^{v}$, Eq. (2.13) becomes the HQET Lagrangian in the rest frame. Although the $1 / m_{a}$ term is naively subleading for small relative momentum, it plays a crucial role in certain circumstances, as we shall see in Sec. IV. Nonetheless, let us state that for the correlators we will be interested in one can safely neglect it and work with the HQET Lagrangian.

$L^{I}$ mixes small and large momenta:

$$
L^{I}=L_{\mu}^{I}+L_{\mu}^{I \mu}
$$

The first term reads

$$
\mathcal{L}_{\mu}^{I}(x)=g \bar{Q}_{a} \gamma^{0} B_{0}^{r} T^{r} Q_{a}(x),
$$

which gives the leading contribution to the multipole expansion. We will not discuss these contributions (2.15) here since they have been extensively studied in the literature $[3,7,8]$. Let us focus on the second term. It reads

$$
\begin{aligned}
L_{\mu}^{I \mu}= & -\frac{1}{2} \sum_{a a^{\prime} A} m_{a a^{\prime}}^{3} N_{A}^{2} \sum_{s} \int \frac{d^{3} v}{(2 \pi)^{3}} \int_{\mu} \frac{d^{3} q^{\prime}}{(2 \pi)^{3}} \int^{\mu} \frac{d^{3} q}{(2 \pi)^{3}} V^{A}\left(\vec{q}^{\prime}-\vec{q}\right)\left[\tilde{\hat{h}_{a}^{v}}(\vec{q}, t) T^{A} \bar{\Gamma}_{s} \widetilde{h_{a}^{v}}(\vec{q}, t)\right] \\
& \times\left[\tilde{Q}_{a^{\prime}}\left(m_{a^{\prime}} \vec{v}+\vec{q}^{\prime}, t\right) T^{A} \Gamma_{s} \widetilde{Q}_{a}\left(-m_{a} \vec{v}+\vec{q}^{\prime}, t\right)\right]+\text { H.c. }
\end{aligned}
$$

In this expression the Coulomb potential is the only piece which mixes small and large relative momenta. We can perform a derivative expansion since $q$ and $q^{\prime}$ belong to different momentum regimes $\left(q \sim \Lambda_{\mathrm{QCD}} \ll q^{\prime} \sim m \alpha / n\right)$ and keep only the leading term (further orders would give subleading corrections). It turns out that the small relative momentum term decouples from the Coulomb potential and can be written like a local current. Finally, we obtain

$$
\begin{aligned}
L_{\mu}^{I \mu}= & -\frac{1}{2} \sum_{a a^{\prime} A} m_{a a^{\prime}}^{3} N_{A}^{2} \sum_{s} \int \frac{d^{3} v}{(2 \pi)^{3}} \int_{\mu} \frac{d^{3} q}{(2 \pi)^{3}} V^{A}(\vec{q}) \int d^{3} x e^{-i m_{a a^{\prime}} \vec{v} \cdot \vec{x}} J_{l, \bar{\Gamma}_{s}}^{A, a a^{\prime}}(\vec{x}, t) \\
& \times\left[\tilde{\tilde{Q}}_{a^{\prime}}\left(m_{a^{\prime}} \vec{v}+\vec{q}, t\right) T^{A} \Gamma_{s} \widetilde{Q}_{a}\left(-m_{a} \vec{v}+\vec{q}, t\right)\right]+\text { H.c. }
\end{aligned}
$$

The formalism developed in [6] was not powerful enough to uncover the interaction Lagrangian (2.17). This interaction Lagrangian is indeed responsible for the differences between the results presented there and the ones obtained in the next section.

If we assume that small momentum terms are small in

\footnotetext{
${ }^{4}$ Several aspects related to this cutoff dependence have been studied in [15].
}

comparison with the large momentum terms, we can treat the interaction Lagrangian (2.17) as a perturbation. This is so for the lower-energy levels of heavy quark bound states. In the next sections we focus on the nonperturbative contributions coming from Eqs. (2.13) and (2.17).

\section{PHYSICAL OBSERVABLES}

In this section we work out the nonperturbative corrections from the small relative momentum region to the decay 
constant, the bound state mass, and the matrix elements of bilinear currents at zero recoil. We take the bound state velocity small or zero.

Consider first the eigenvalues and eigenstates of $H_{\mu}$, the Hamiltonian associated with $L_{\mu}$. They read

$$
\begin{aligned}
&|(a b, n, s, A) ; \vec{v}\rangle= \frac{N_{A}}{\sqrt{2}} \int_{\mu} \frac{d^{3} k}{(2 \pi)^{3}} \widetilde{\Phi}_{a b, n}^{A}(\vec{k} ; \mu) \vec{u}^{\alpha}\left(\vec{p}_{1}\right) \\
& \times \Gamma_{s} v^{\beta}\left(\vec{p}_{2}\right) T_{i_{1}, i_{2}}^{A} b_{\alpha, i_{1}}^{a \dagger}\left(\vec{p}_{1}\right) d_{\beta, i_{2}}^{b \dagger}\left(\vec{p}_{2}\right)|0\rangle \\
& E_{a b, n}^{A}(\mu)
\end{aligned}
$$

where

$$
\vec{p}_{1}=m_{a} \vec{v}+\vec{k}, \quad \vec{p}_{2}=m_{b} \vec{v}-\vec{k}
$$

$E_{a b, n}^{A}, \Phi_{a b, n}^{A}(\vec{x})$, and $\widetilde{\Phi}_{a b, n}^{A}(\vec{k})$ are the energy, the coordinate space wave function, and the momentum space wave function of a Coulomb-type state with quantum number $n=(n, l, m)$, respectively. $\vec{v}$ is the bound state three-vector velocity. $a$ and $b$ are flavor indices and $s$ denotes spin. $b^{\dagger}$ and $d^{\dagger}$ are creation operators of particles and antiparticles, respectively:

$$
\begin{gathered}
\left\{b_{\alpha, i_{1}}^{a \dagger}\left(\vec{p}_{1}\right), b_{\beta, i_{2}}^{b}\left(\vec{p}_{2}\right)\right\}=(2 \pi)^{3} \delta^{a b} \delta_{\alpha \beta} \delta_{i_{1} i_{2}} \delta^{3}\left(\vec{p}_{1}-p_{2}\right), \\
\left\{d_{\alpha, i_{1}}^{a \dagger}\left(\vec{p}_{1}\right), d_{\beta, i_{2}}^{b}\left(\vec{p}_{2}\right)\right\}=(2 \pi)^{3} \delta^{a b} \delta_{\alpha \beta} \delta_{i_{1} i_{2}} \delta^{3}\left(\vec{p}_{1}-\vec{p}_{2}\right), \\
\left\{b_{\alpha, i_{1}}^{a \dagger}\left(\vec{p}_{1}\right), d_{\beta, i_{2}}^{b}\left(\vec{p}_{2}\right)\right\}=0 .
\end{gathered}
$$

$u^{\alpha}\left(\vec{p}_{1}\right)$ and $v^{\beta}\left(\vec{p}_{2}\right)$ are spinors normalized in such a way that in the large- $m$ limit the following holds:

$$
\sum_{\alpha} u^{\alpha}\left(\vec{p}_{1}\right) \vec{u}^{\alpha}\left(\vec{p}_{1}\right)=p_{+}, \quad \sum_{\alpha} v^{\alpha}\left(\vec{p}_{1}\right) \vec{v}^{\alpha}\left(\vec{p}_{1}\right)=-p_{-}
$$

Equation (3.1) has the nonrelativistic normalization

$$
\begin{aligned}
& \left\langle(a b, n, s, A) ; \vec{v} \mid\left(a^{\prime} b^{\prime}, n^{\prime}, s^{\prime}, A^{\prime}\right) ; \vec{v}^{\prime}\right\rangle \\
& =(2 \pi)^{3} \delta^{(3)}\left(m_{a b}\left(\vec{v}-\vec{v}^{\prime}\right)\right) \delta_{n, n^{\prime}} \delta_{s, s^{\prime}} \\
& \quad \times \delta_{(a b),\left(a^{\prime} b^{\prime}\right)} \delta_{A, A^{\prime}},
\end{aligned}
$$

where we have used

$$
\operatorname{tr}\left(p_{+} \Gamma^{s} p_{-} \bar{\Gamma}^{s^{\prime}}\right)=-2 \delta_{s, s^{\prime}} .
$$

From Eq. (3.8) the wave function normalization follows:

$$
\int_{\mu} \frac{d^{3} q}{(2 \pi)} \widetilde{\Phi}_{a b, n^{\prime}}^{A *}(\vec{q} ; \mu) \widetilde{\Phi}_{a b, n}^{A}(\vec{q} ; \mu)=\delta_{n, n^{\prime}},
$$

where there is no sum over $A$. The wave function and the energy fulfill the equation

$$
\begin{gathered}
\frac{p^{2}}{2 \mu_{a b}} \widetilde{\Phi}_{a b, n}^{A}(\vec{p} ; \mu)+\int_{\mu} \frac{d^{3} q}{(2 \pi)^{3}} \widetilde{\Phi}_{a b, n}^{A}(\vec{q} ; \mu) V^{A}(\vec{p}-\vec{q}) \\
=E_{a b, n}^{A}(\mu) \widetilde{\Phi}_{a b, n}^{A}(\vec{p} ; \mu), \\
p>\mu, \quad \mu_{a b}=\frac{m_{a} m_{b}}{m_{a}+m_{b}} .
\end{gathered}
$$

From Eq. (2.8) it trivially follows that the eight components of the octet wave function fulfill the same equation and hence they are the same. Notice that the wave function normalization and the differential equation above are $\mu$ dependent. Furthermore, the wave function is not defined over all values of $p$. We will work this out in detail in Sec. IV. In order to simplify the notation we will not display the cutoff dependence explicitly in the rest of the section, but it must be understood throughout.

For $H^{\mu}$, the Hamiltonian associated with $L^{\mu}$, we denote the eigenstates and eigenvalues by

$$
|(a b, g, s) ; \vec{v}\rangle, \quad E_{g},
$$

where $g$ labels the low-momentum state. We cannot give explicit expressions since their dynamics is governed by low momentum. Equation (3.12) has the nonrelativistic normalization

$$
\begin{aligned}
& \left\langle(a b, g, s) ; \vec{v} \mid\left(a^{\prime} b^{\prime}, g^{\prime}, s^{\prime}\right) ; \vec{v}^{\prime}\right\rangle \\
& \quad=(2 \pi)^{3} \delta^{(3)}\left(m_{a b}\left(\vec{v}-\vec{v}^{\prime}\right)\right) \delta_{s, s^{\prime}} \delta_{(a b),\left(a^{\prime} b^{\prime}\right)} \delta_{g, g^{\prime}}
\end{aligned}
$$

Of course, the states (3.1) and (3.12) are orthogonal since they belong to different momentum regimes.

Our Hilbert space is (before switching on $L_{\mu}^{I \mu}$ ) $\{N\}$ $=\{(n, A), g\}$ and the identity reads, in this base,

$$
\begin{aligned}
1 & \simeq|0\rangle\langle 0|+1^{\mu}+1_{\mu} \\
& =|0\rangle\left\langle 0\left|+\sum_{a b, N, s} \int \frac{d^{3} \vec{P}}{(2 \pi)^{3}}\right|(a b, N, s) ; \vec{v}\right\rangle\langle(a b, N, s) ; \vec{v}| .
\end{aligned}
$$

Let us now calculate the matrix elements of $H_{\mu}^{I \mu}$, the Hamiltonian associated with $L_{\mu}^{I \mu}$. We note that the only matrix element different from zero is

$$
\begin{aligned}
& \left\langle(a b, g, s) ; \vec{v}\left|H_{\mu}^{I \mu}\right|\left(a^{\prime} b^{\prime}, n, s^{\prime}, A\right) ; \vec{v}^{\prime}\right\rangle \\
& =(2 \pi)^{3} \delta^{(3)}\left(m_{a b}\left(\vec{v}-\vec{v}^{\prime}\right)\right) E_{a b, n^{\prime}}^{A}, \widetilde{\Phi}_{a b, n^{\prime}}^{A}(0) \\
& \quad \times \frac{f_{a b, g}^{A *} N_{A}}{\sqrt{2}} \delta_{s, s^{\prime}} \delta_{(a b),\left(a^{\prime} b^{\prime}\right)}
\end{aligned}
$$

where

$$
\left\langle(a b, g, s) ; \vec{v}\left|\overline{h_{a}^{v}} T^{A} \Gamma^{s^{\prime}} h_{b}^{v}(0)\right| 0\right\rangle=: f_{a b, g}^{A *} \delta_{s, s^{\prime}} .
$$

In the calculations above we have not made any explicit assumption about the relative size of $L_{\mu}$ and $L^{\mu}$. We are mainly interested in very heavy quark-antiquark bound states where small momenta can be considered as corrections, at 
least for the lower-energy levels. Clearly, these bound states should be singlets since the octet potential is repulsive. In fact, at the level we are working, the octet states are not going to give contributions to the physical observables, and so we will neglect them in the following. Hence from now on color singlets are understood and color indeces dropped. We also remark that we are always working in the c.m. frame, even though sometimes we keep $\vec{v} \neq 0$ in some intermediate steps for convenience. Following standard quantum mechanics perturbation theory [17] we can obtain the corrected bound state energy ${ }^{5}$ and wave functions (states) for the lower levels. They read

$$
\begin{aligned}
& \delta E_{a b, n}=\frac{\Pi_{a b}\left(E_{a b, n}\right)}{2 N_{c}}\left|E_{a b, n} \widetilde{\Phi}_{a b, n}(\overrightarrow{0})\right|^{2}, \\
& |(a b, n, s) ; \vec{v}\rangle_{F}=Z_{n}^{1 / 2}|\overline{(a b, n, s) ; \vec{v}}\rangle_{F}, \\
& |\overrightarrow{(a b, n, s) ; \vec{v}}\rangle_{F}=|(a b, n, s) ; \vec{v}\rangle+|(a b, n, s) ; \vec{v}\rangle^{(1)} \\
& +|(a b, n, s) ; \vec{v}\rangle^{(2)}+\cdots, \\
& |(a b, n, s) ; \vec{v}\rangle^{(1)}=\frac{\widetilde{\Phi}_{a b, n}(\overrightarrow{0}) E_{a b, n}}{\sqrt{2 N_{c}}} \hat{G}^{\mu}\left(E_{a b, n}\right) J_{l, \Gamma^{s}}^{a b}(0)|0\rangle, \\
& |(a b, n, s) ; \vec{v}\rangle^{(2)}=\sum_{m \neq n}|(a b, m, s) ; \vec{v}\rangle \frac{\Pi_{a b}\left(E_{a b, n}\right)}{2 N_{c}} \\
& \times E_{a b, n} \widetilde{\Phi}_{a b, m}^{*}(\overrightarrow{0}) \widetilde{\Phi}_{a b, n}(\overrightarrow{0}) \frac{E_{a b, m}}{E_{a b, n}-E_{a b, m}}, \\
& Z_{n} \simeq 1+\frac{1}{2 N_{c}} \frac{d \Pi_{a b}\left(E_{a b, n}\right)}{d E_{a b, n}}\left|E_{a b, n} \widetilde{\Phi}_{a b, n}(\overrightarrow{0})\right|^{2}
\end{aligned}
$$

where both continuum and bound states are included in the sum in Eq. (3.21), Eq. (3.18) denotes the physical normalized state (with low-momentum corrections), and

$$
\begin{gathered}
\hat{G}^{\mu}(z):=\frac{1}{z-H^{\mu}+i \epsilon}, \\
i \int d^{4} x e^{i P_{n^{x}}\left\langle 0\left|T\left\{J_{l, \bar{\Gamma}^{s^{\prime}}}^{b a}(x) J_{l, \Gamma^{s}}^{a b}(0)\right\}\right| 0\right\rangle:} \\
=: \Pi_{a b}\left(E_{a b, n}\right) \operatorname{tr}\left(\bar{\Gamma}^{s^{\prime}} \Gamma^{s}\right), \\
P_{n}^{a b}=\left(m_{a b, n}, 0\right), \quad m_{a b, n}:=m_{a b}+E_{a b, n} .
\end{gathered}
$$

We should stress that in the last two equations there is only small momentum dynamics. High energies may come from the external bound state energy insertion.

Some comments are in order. Notice first that for $l \neq 0$ (angular momentum) the wave function (state) and the energy remain unchanged. Notice also that the $s$-wave state does not receive contributions from $l \neq 0$ states either. The previous statement is true because of the fact that the momentum wave function at zero momentum for $l \neq 0$ is zero. This means that the new interaction does not couple $l=0$ states with $l \neq 0$ states. This result would change if we kept further terms in the effective Lagrangian [see Eq. (2.16)] but, of course, these contributions would be subleading.

Let us next calculate the decay constant. In order to do it we split the current as in the last section. The soft current only gives a contribution with the low-momentum states $g$ in the same way as the hard current only gives a contribution with the modified Coulomb bound states. Thus, we obtain

$$
\begin{gathered}
\left\langle 0\left|J_{\Gamma}^{b a}(0)\right|(a b, g, s) ; \vec{v}\right\rangle=\left\langle 0\left|J_{l, \Gamma}^{b a}(0)\right|(a b, g, s) ; \vec{v}\right\rangle=-\frac{\operatorname{tr}\left(\Gamma^{s} \Gamma\right)}{2} f_{a b, g}, \\
\left\langle 0\left|J_{\Gamma}^{b a}(0)\right|(a b, n, s) ; \vec{v}\right\rangle=\left\langle 0\left|J_{h, \Gamma}^{b a}(0)\right|(a b, n, s) ; \vec{v}\right\rangle=-\operatorname{tr}\left(\Gamma^{s} \Gamma\right) \sqrt{\frac{N_{c}}{2}} \Phi_{a b, n}(\overrightarrow{0}) .
\end{gathered}
$$

Finally the decay constant reads (changing to relativistic normalization)

$$
\begin{aligned}
\left\langle 0\left|J_{\Gamma}^{b a}(0)\right|(a b, n, s) ; \vec{v}\right\rangle_{F}= & -\operatorname{tr}\left(\Gamma^{s} \Gamma\right) \sqrt{m_{a b, n} N_{c}} \Phi_{a b, n}(\overrightarrow{0})\left\{1+\frac{1}{4 N_{c}} \frac{d \Pi_{a b}\left(E_{a b, n}\right)}{d E_{a b, n}}\left|E_{a b, n} \widetilde{\Phi}_{a b, n}(\overrightarrow{0})\right| 2\right. \\
& +\frac{\Pi_{a b}\left(E_{a b, n}\right)}{2 N_{c}} E_{a b, n} \frac{\widetilde{\Phi}_{a b, n}(\overrightarrow{0})}{\Phi_{a b, n}(\overrightarrow{0})}\left(1+\sum_{m \neq n} \Phi_{a b, m}(\overrightarrow{0}) \widetilde{\Phi}_{a b, m}^{*}(\overrightarrow{0}) \frac{E_{a b, m}}{E_{a b, n}-E_{a b, m}}\right) .
\end{aligned}
$$

Finally let us obtain the bilinear currents at zero recoil. For that we need to know

\footnotetext{
${ }^{5}$ The correction to the bound state energy was found to be zero in [6] because the existence of $L_{\mu}^{I \mu}$ was not known.
} 


$$
\begin{gathered}
J_{\Gamma}^{b c}(0)=\bar{Q}^{b} \Gamma Q^{c}(0), \\
\left\langle\left(a c, N^{\prime}, s^{\prime}\right) ; \vec{v}\left|J_{\Gamma}^{b c}(0)\right|(a b, N, s) ; \vec{v}\right\rangle .
\end{gathered}
$$

In order to deal with them we need to perform the splitting between large and small momenta. However, this current cannot be in general split into two terms. We have mixing between large and small momenta. Fortunately, the mixing terms disappear if both initial and final states have the same velocity. This will not longer be true for non-zero recoil matrix elements. Thus, we obtain

$$
\begin{gathered}
\left\langle\left(a c, n^{\prime}, s^{\prime}\right) ; \vec{v}\left|J_{\Gamma}^{b c}(0)\right|(a b, n, s) ; \vec{v}\right\rangle=-\frac{\operatorname{tr}\left(\bar{\Gamma}^{s^{\prime}} \Gamma^{s} \Gamma\right)}{2} \int_{\mu} \frac{d^{3} \vec{k}}{(2 \pi)^{3}} \widetilde{\Phi}_{a c, n^{\prime}}^{*}(\vec{k}) \widetilde{\Phi}_{a b, n}(\vec{k}), \\
\left\langle\left(a c, g^{\prime}, s^{\prime}\right) ; \vec{v}\left|J_{\Gamma}^{b c}(0)\right|(a b, g, s) ; \vec{v}\right\rangle=:-\frac{\operatorname{tr}\left(\bar{\Gamma}^{s^{\prime}} \Gamma^{s} \Gamma\right)}{2} f_{a c, a b}^{g^{\prime} g}
\end{gathered}
$$

where we have used

$$
\sum_{s}\left(\Gamma^{s}\right)_{\alpha_{2} \alpha_{4}}\left(\bar{\Gamma}^{s}\right)_{\alpha_{1} \alpha_{3}}=-2\left(p_{+}\right)_{\alpha_{2} \alpha_{3}}\left(p_{-}\right)_{\alpha_{1} \alpha_{4}} .
$$

The remaining possible matrix elements are zero. Notice that $f_{a b, a b}^{g^{\prime} g}=\delta_{g^{\prime} g}$ because of baryonic charge conservation.

The physical matrix element reads (again with relativistic normalization)

$$
\begin{aligned}
{ }_{F}\left\langle\left(a c, n^{\prime}, s^{\prime}\right) ; \vec{v}\left|J_{\Gamma}^{b c}(0)\right|(a b, n, s) ; \vec{v}\right\rangle_{F}= & -\sqrt{m_{a b, n} m_{a c, n^{\prime}}} \operatorname{tr}\left(\bar{\Gamma}^{s^{\prime}} \Gamma^{s} \Gamma\right)\left\{\int_{\mu} \frac{d^{3} \vec{k}}{(2 \pi)^{3}} \widetilde{\Phi}_{a c, n^{\prime}}^{*}(\vec{k}) \widetilde{\Phi}_{a b, n}(\vec{k})\{1\right. \\
& \left.+\frac{1}{4 N_{c}} \frac{d \Pi_{a b}\left(E_{a b, n}\right)}{d E_{a b, n}}\left|E_{a b, n} \widetilde{\Phi}_{a b, n}(\overrightarrow{0})\right| 2+\frac{1}{4 N_{c}} \frac{d \Pi_{a c}\left(E_{a c, n^{\prime}}\right)}{d E_{a c, n^{\prime}}}\left|E_{a c, n^{\prime}} \widetilde{\Phi}_{a c, n^{\prime}}(\overrightarrow{0})\right| 2\right\} \\
& +\frac{\Pi_{a c, a b}\left(E_{a c, n^{\prime}}, E_{a b, n}\right)}{2 N_{c}} E_{a c, n^{\prime}} E_{a b, n} \widetilde{\Phi}_{a c, n^{\prime}}^{*}(\overrightarrow{0}) \widetilde{\Phi}_{a b, n}(\overrightarrow{0}) \\
& +\frac{\Pi_{a c}\left(E_{a c, n^{\prime}}\right)}{2 N_{c}} E_{a c, n^{\prime}} \sum_{m \neq n^{\prime}} \widetilde{\Phi}_{a c, m}(\overrightarrow{0}) \widetilde{\Phi}_{a c, n^{\prime}}^{*}(\overrightarrow{0}) \frac{E_{a c, m}}{E_{a c, n^{\prime}}-E_{a c, m}} \\
& \times \int_{\mu} \frac{d^{3} \vec{k}}{(2 \pi)^{3}} \widetilde{\Phi}_{a c, m}^{*}(\vec{k}) \widetilde{\Phi}_{a b, n}(\vec{k})+\frac{\Pi_{a b}\left(E_{a b, n}\right)}{2 N_{c}} E_{a b, n} \sum_{m \neq n} \widetilde{\Phi}_{a b, m}^{*}(\overrightarrow{0}) \\
& \left.\times \widetilde{\Phi}_{a b, n}(\overrightarrow{0}) \frac{E_{a b, m}}{E_{a b, n}-E_{a b, m}} \int_{\mu} \frac{d^{3} \vec{k}}{(2 \pi)^{3}} \widetilde{\Phi}_{a c, n^{\prime}}^{*}(\vec{k}) \widetilde{\Phi}_{a b, m}(\vec{k})\right\},
\end{aligned}
$$

where

$$
\int d^{4} x_{1} d^{4} x_{2} e^{i P_{n^{\prime}}^{a c} x_{1}} e^{-i P_{n}^{a b} x_{2}}\left\langle 0\left|T\left\{J_{l, \bar{\Gamma}^{s^{\prime}}}^{c a}\left(x_{1}\right) J_{l, \Gamma}^{b c}(0) J_{l, \Gamma^{s}}^{a b}\left(x_{2}\right)\right\}\right| 0\right\rangle=: \Pi_{a c, a b}\left(E_{a c, n^{\prime}}, E_{a b, n}\right) \operatorname{tr}\left(\bar{\Gamma}^{s^{\prime}} \Gamma^{s} \Gamma\right) .
$$

We can easily check that orthonormality is satisfied ${ }^{6}$ when $b=c$. We expect the last statement to be true since spin symmetry relates the matrix element with the baryonic charge when $b=c$ :

$$
{ }_{F}\left\langle\left(a b, n^{\prime}, s^{\prime}\right) ; \vec{v}\left|J_{\Gamma}^{b b}(0)\right|(a b, n, s) ; \vec{v}\right\rangle_{F}=-m_{a b, n} \operatorname{tr}\left(\bar{\Gamma}^{s^{\prime}} \Gamma^{s} \Gamma\right) \delta_{n, n^{\prime}} .
$$

Before finishing this section let us make some remarks. Both correlators (3.24) and (3.34) should be small quantities for

${ }^{6}$ This was not always the case for the result given in [6]. perturbation theory to hold. This is the case if $\mu$ is small against the typical momentum in the Coulomb interaction (i.e., $\mu a_{a b, n} \ll 1$, where $a_{a b, n}=n / \mu_{a b} \alpha$ is the Bohr radius). It constrains the possible applications to the lower-energy levels. On the other hand, $\Lambda_{\mathrm{QCD}} \ll \mu$ should hold so that the low-momentum dynamics is not strongly affected by the cutoff. 


\section{CUTOFF INDEPENDENCE}

Our results in the last section may look strongly cutoff dependent. We have two sources of cutoff dependence. On the one hand, we have a cutoff separating small momentum gluons from large momentum gluons. This cutoff is the responsible for the absence of a Coulomb interaction in $L^{\mu}$. It has been mentioned at several instances but it has never been written down explicitly in the formulas. This cutoff dependence has been analyzed before [15] and so we shall ignore it in the following. On the other hand, we have the cutoff separating large and small relative momenta. It plays the role of an infrared cutoff in the perturbative Coulomb wave function (large momentum) and the role of an ultraviolet cutoff for the small momentum contributions. We prove in this section the cutoff independence to the desired order $\left(\mu^{3}, \Lambda_{\mathrm{QCD}}^{3}\right)$ of this last cutoff. This is crucial to ensure that our approach respects color SU (3) gauge symmetry. It is important to use the same cutoff procedure in both large and small momentum regions in order to neatly cancel the cutoff dependence. We use a hard three-momentum cutoff for convenience, as we have done in the previous sections.

First of all, let us study the cutoff dependence in the lowmomentum correlators we found in the last section. Although they are nonperturbative objects, we can always perform a perturbative calculation in order to see how they depend on the cutoff.

Let us start by with Eq. (2.13) (which is formally equal to NRQCD). For Eq. (3.24) we obtain, at the lowest order (in the c.m. frame, $\vec{v}=0$ ),

$$
\begin{gathered}
\Pi_{a b}\left(k^{0}\right)=-\frac{N_{c} \mu_{a b} \mu}{\pi^{2}}\left[1-\frac{1}{2 x} \ln \left(\frac{1+x}{1-x}\right)\right], \\
x=\frac{\mu}{\sqrt{2 \mu_{a b}\left(k^{0}+i \epsilon\right)}} .
\end{gathered}
$$

Let us consider two limits.

In the limit $x \gg 1$ (i.e., near threshold) it reduces to

$$
\Pi_{a b}\left(k^{0}\right) \simeq-\frac{N_{c} \mu_{a b} \mu}{\pi^{2}}\left[1+\frac{i \pi}{2 x} \theta\left(k^{0}\right)\right]
$$

and no pole appears. In the limit $x \ll 1$, Eq. (4.1) reduces to

$$
\Pi_{a b}\left(k^{0}\right) \simeq \frac{N_{c} \mu^{3}}{6 \pi^{2}} \frac{1}{\left(k^{0}+i \epsilon\right)} .
$$

This expression is going to be important in the following. We stress that Eq. (4.3) is $\mu_{a b}$ independent, and amounts to drop the $1 / m$ terms in Eq. (2.13) which is nothing but the HQET for quarks and antiquarks. Let us now look for the physical situation we are interested in. Thus, we take $k^{0}=E_{a b, n}^{0}$ and we obtain $|x|=\mu n / \mu_{a b} C_{F} \alpha_{s}$, but this is nothing but the parameter we need to keep small so that the small relative momentum contributions are subleading, and hence our expansion makes sense. In the following, we always consider that we are in the limit $|x| \ll 1$.

For Eq. (3.34) we obtain $(x \ll 1)$

$$
\Pi_{a c, a b}\left(k^{\prime 0}, k^{0}\right) \simeq \frac{N_{c} \mu^{3}}{6 \pi^{2}} \frac{1}{\left(k^{0}+i \epsilon\right)} \frac{1}{\left(k^{\prime 0}+i \epsilon\right)} .
$$

At this point we would like to stress that in the limit $x \ll 1$ the same perturbative results are found using HQET. This is going to be determinant in the next section.

Equations (4.3) and (4.4) make explicit the UV cutoff dependence coming from the small relative momentum region. Let us next go on with the IR cutoff dependence coming from the large relative momentum region.

Let us then study the cutoff dependence of the wave function (for simplicity we omit the flavor indices). In order to do it we solve the wave equation (3.11) perturbatively in $\mu . n$ labels a continuum or discrete spectrum. Because of the radial symmetry, we can write (we follow [18])

$$
\widetilde{\Phi}_{n, l, m}(\vec{p} ; \mu)=F_{n, l}(p ; \mu) Y_{l, m}(\hat{p}),
$$

where $F_{n, l}(p ; \mu)$ satisfies

$$
\begin{gathered}
\frac{p^{2}}{2 \mu_{a b}} F_{n, l}(p ; \mu)-\frac{C_{F} \alpha}{\pi p} \int_{\mu} q d q F_{n, l}(q ; \mu) Q_{l}\left(\frac{p^{2}+q^{2}}{2 p q}\right) \\
=E_{n}(\mu) F_{n, l}(p ; \mu), \quad p>\mu \\
Q_{l}(z)=\frac{1}{2} \int_{-1}^{1} d x \frac{P_{l}(x)}{z-x}
\end{gathered}
$$

and $P_{l}$ is the Legendre function of the first kind. We stress that we are interested in $F_{n, l}(p ; \mu)$ for $p>\mu$ only, although in the intermediate steps it is going to be defined over all $p>0$ values. Now we perform a cutoff parameter expansion and we work as in the usual quantum mechanics perturbation theory where we demand the corrections to be orthogonal to the leading result:

$$
F_{n, l}(p ; \mu)=\sum_{r=0}^{\infty} F_{n, l}^{r}(p) \frac{\mu^{r}}{r !}, \quad E_{n}=\sum_{r=0}^{\infty} E_{n}^{r} \frac{\mu^{r}}{r !} .
$$

We also expand the cutoff in the integral:

$$
\int_{\mu} q d q F_{n, l}^{r}(q) Q_{l}\left(\frac{p^{2}+q^{2}}{2 p q}\right) \equiv h_{n, l}^{r}(p, \mu) \equiv \sum_{i=0}^{\infty} h_{n, l}^{r, i}(p) \frac{\mu^{i}}{i !} .
$$

On general grounds we can see that the corrections to the Coulomb wave function and energy go like $O\left(\mu^{2 l+3}\right)$; therefore, as expected, we can neglect the $l \neq 0$ states since their contributions are subleading.

At leading order we obtain the standard Schrödinger equation with a Coulomb potential with no $\mu$ dependence. Furthermore, for the following terms in perturbation theory we obtain

$$
E_{n}^{1}=E_{n}^{2}=0, \quad \widetilde{\Phi}_{n}^{1}(\vec{p})=\widetilde{\Phi}_{n}^{2}(\vec{p})=0 .
$$

Finally to third order we obtain

$$
E_{n}^{3}=-E_{n} \frac{\left|\widetilde{\Phi}_{n}(\overrightarrow{0})\right|^{2}}{\pi^{2}}
$$




$$
\widetilde{\Phi}_{n}^{3}(\vec{p})=\sum_{m \neq n} \widetilde{\Phi}_{m}(\vec{p}) \frac{\widetilde{\Phi}_{m}^{*}(\overrightarrow{0}) \widetilde{\Phi}_{n}(\overrightarrow{0})}{\pi^{2}} \frac{E_{m}}{E_{m}-E_{n}} .
$$

We have not yet normalized the cutoff-dependent wave function, as we can see from

$$
\int_{\mu} \frac{d^{3} q}{(2 \pi)^{3}} \widetilde{\Phi}_{n}^{*}(\vec{q}) \widetilde{\Phi}_{n}(\vec{q}) \simeq 1-\left|\widetilde{\Phi}_{n}(\overrightarrow{0})\right|^{2} \frac{\mu^{3}}{6 \pi^{2}} .
$$

Therefore, we must change

$$
\widetilde{\Phi}_{n}(\vec{p} ; \mu) \rightarrow \widetilde{\Phi}_{n}(\vec{p} ; \mu)\left(1+\left|\widetilde{\Phi}_{n}(\overrightarrow{0})\right|^{2} \frac{\mu^{3}}{12 \pi^{2}}\right) .
$$

Equations (4.11)-(4.14) provide the explicit $I R$ cutoff dependence from the large relative momentum region.

We have obtained the explicit cutoff dependence to the desired order $\mu^{3}$ in both large and small momentum regions. Now we will see they match properly, that is, the observables are cutoff independent. In fact, what we will see is that the physical states (3.18) themselves are already cutoff independent. In this way we prove the cutoff independence for any observable.

Consider first the bound state energy

$$
E_{n}^{F}=E_{n}+\delta E_{n} .
$$

The cutoff dependence of $E_{n}$ is given by Eqs. (4.8), (4.10), and (4.11), whereas the cutoff dependence of $\delta E_{n}$ is given by Eqs. (3.17) and (4.3). One can then easily check that $E_{n}^{F}$ is cutoff independent.

Consider next the state $|\overline{(a b, n, s) ; \vec{v}}\rangle_{F}$ in Eq. (3.19). Recall that the first and last terms on the right-hand side (RHS) belong to the large relative momentum region whereas the term in the middle belongs to small relative momentum region. Let us keep apart for a moment the explicit cutoff separating these two regions in the relative momentum integrals. The remaining cutoff dependences of the first term are given by Eqs. (4.5), (4.8), (4.10), and (4.12), while for the last term are given by Eqs. (3.21) and (4.3), which cancel each other.

It remains the $U V$ cutoff dependence coming from Eq. (3.20) (which has been already studied in [6]) and the explicit IR cutoff dependence coming from the integral over relative momentum in the first term of Eq. (3.19) [see Eq. (3.1)], which we kept apart for a while. Recall that the wave function in the first term of Eq. (3.19) is, except for the normalization factor (4.14), the Coulomb wave function since we have already canceled the cutoff dependences coming from Eq. (4.12). Let us next calculate Eq. (3.20) perturbatively at lowest order. It reads

$$
\begin{aligned}
|(a b, n, s) ; \vec{v}\rangle^{(1)}= & \frac{\widetilde{\Phi}_{a b, n}(\overrightarrow{0})}{\sqrt{2 N_{c}}} \int^{\mu} \frac{d^{3} \vec{k}}{(2 \pi)^{3}} \vec{u}^{\alpha}\left(p_{1}\right) \\
& \times \Gamma_{s} v^{\beta}\left(p_{2}\right) b_{\alpha, i}^{a \dagger}\left(p_{1}\right) d_{\beta, i}^{b \dagger}\left(p_{2}\right)|0\rangle \\
= & \frac{1}{\sqrt{2 N_{c}}} \int^{\mu} \frac{d^{3} \vec{k}}{(2 \pi)^{3}} \widetilde{\Phi}_{a b, n}(\vec{k}) \vec{u}^{\alpha}\left(p_{1}\right) \\
& \times \Gamma_{s} v^{\beta}\left(p_{2}\right) b_{\alpha, i}^{a \dagger}\left(p_{1}\right) d_{\beta, i}^{b \dagger}\left(p_{2}\right)|0\rangle .
\end{aligned}
$$

The second equality holds at the order we are working at. Notice finally that this is nothing but the piece we need to add to the first term of Eq. (3.19) in order to obtain a relative momentum integral independent of the cutoff. Finally, the cutoff dependences of the normalization in Eq. (4.14) and of Eq. (3.22) also cancel each other in Eq. (3.18) [again taking into account Eq. (4.3)].

We have thus seen that at the level of physical states we are able to prove the cutoff independence. The cutoff independence can also be checked explicitly in the observables (3.17), (3.27), and (3.33). This demonstrates that the HQET ultraviolet behavior cancels the NRQCD infrared behavior in Coulomb-type bound states, which guarantees that we have performed a proper matching between large and small relative momenta. This issue has also been pursued in $[6,15]$.

\section{EVALUATION OF THE LOW-MOMENTUM CORRELATORS}

In Sec. III we learned how to parametrize the possible nonperturbative contributions in the small relative momentum region in terms of two low-momentum correlators [Eqs. (3.24) and (3.34)] with external Coulomb bound state energy insertions. It is remarkable that these contributions only exist for $s$ states. At the beginning of Sec. IV we also saw that the kinetic term, which is suppressed by a mass invers power, can be safely neglected in the correlators we are interested in, and hence we can use HQET for quarks and antiquarks to discuss their properties.

The HQET for quarks and antiquarks enjoys a $\mathrm{U}\left(4 N_{h f}\right)$ symmetry which breaks spontaneously down to the $\mathrm{U}\left(2 N_{h f}\right) \otimes \mathrm{U}\left(2 N_{h f}\right)$ Isgur-Wise symmetry [12].

Let us first analyze the consequences of the unbroken $\mathrm{U}\left(2 N_{h f}\right) \otimes \mathrm{U}\left(2 N_{h f}\right)$ symmetry. In fact, the spin symmetry which is included in it has already been used in Eqs. (3.24) and (3.34). The flavor symmetry implies

$$
f_{a b, g}=f_{g}, \quad f_{a c, a b}^{g^{\prime} g}=f^{g^{\prime} g} .
$$

Therefore we get

$$
\begin{gathered}
\Pi_{a b}\left(E_{a b, n}\right)=\Pi_{1}\left(E_{a b, n}\right), \\
\Pi_{a c, a b}\left(E_{a c, n^{\prime}}, E_{a b, n}\right)=\Pi_{2}\left(E_{a c, n^{\prime}}, E_{a b, n}\right) .
\end{gathered}
$$

The correlators (3.24) and (3.34) are thus given in terms of two unknown universal (flavor-independent) functions $\Pi_{1}$ and $\Pi_{2}$. But if we go further, using flavor number conservation together with flavor symmetry, we obtain $f^{g^{\prime} g}=\delta_{g^{\prime} g}$ 
for any flavor. From that it follows that if $\Pi_{1}$ is known for any energy insertion, we can obtain $\Pi_{2}$. Explicitly they read

$$
\begin{gathered}
\Pi_{1}\left(E_{a b, n}\right)=\sum_{g} \frac{\left|f_{g}\right|^{2}}{2} \frac{1}{E_{a b, n}-E_{g}}, \\
\Pi^{2}\left(E_{a c, n^{\prime}}, E_{a b, n}\right)=\sum_{g} \frac{\left|f_{g}\right|^{2}}{2} \frac{1}{E_{a c, n^{\prime}}-E_{g}} \frac{1}{E_{a b, n}-E_{g}} .
\end{gathered}
$$

These low-momentum correlators can be further specified at least in two situations: (i) $E_{a b, n} \gg \Lambda_{\mathrm{QCD}}\left(m_{Q} \rightarrow \infty, \alpha\right.$ small); (ii) $E_{a b, n} \ll \Lambda_{\mathrm{QCD}}\left(m_{Q}\right.$ large, $\left.\alpha \rightarrow 0\right)$. Notice that situation (ii) is conceivable if $\alpha$ is very small since so far we have only assumed that the inverse Bohr radius is much larger than $\Lambda_{\mathrm{OCD}}$ and the energy is suppressed by a factor $\alpha$ with respect to the former. ${ }^{7,8}$

In situation (i) the operator product expansion holds. If we carry it out for the low-momentum correlators, we just obtain Eqs. (4.3) and (4.4). Their cutoff dependence just cancels the cutoff dependence from the large relative momentum region, as we saw in Sec. IV. Hence, we conclude that there are no new nonperturbative contributions in this situation, thus confirming the fact that the VL contributions from the condensate are indeed the leading nonperturbative effects in the $m_{Q} \rightarrow \infty$ limit. ${ }^{9}$ This result follows from the observation that there is no local gauge-invariant object that can be built out of $D_{0}$ alone. We have explicitly checked it for lower-order terms.

In situation (ii) we are in the low-energy regime of the HQET. In this regime it is important that the HQET with quarks and antiquarks with the same velocity undergo a spontaneous symmetry breaking of a $\mathrm{U}\left(4 N_{h f}\right)$ symmetry down to the Isgur-Wise symmetry $\mathrm{U}\left(2 N_{h f}\right) \otimes \mathrm{U}\left(2 N_{h f}\right)$, since the Goldstone modes associated with the broken generators dominate the dynamics. The heavy quark hadronic effective Lagrangian describing the Goldstone modes was worked out in [6], where the correlators (3.24) and (3.34) were also calculated. Using those results we obtain

$$
\begin{gathered}
\Pi_{a b}\left(k^{0}\right)=\frac{f_{H}^{2}}{2} \frac{1}{\left(k^{0}+i \epsilon\right)}, \\
\Pi_{a c, a b}\left(k^{\prime 0}, k^{0}\right)=\frac{f_{H}^{2}}{2} \frac{1}{\left(k^{0}+i \epsilon\right)} \frac{1}{\left(k^{\prime 0}+i \epsilon\right)},
\end{gathered}
$$

\footnotetext{
${ }^{7}$ In practice we must remember that $\alpha$ should better be substituted by the running coupling constant at the quarkonium scale, which is in fact an implicit function of $m_{Q}$ and $\Lambda_{\mathrm{QCD}}$.

${ }^{8}$ In [6], the bound state energy $E_{a b, n}$ was understood as giving rise to a residual mass for the heavy quark and antiquark in the heavy quark effective Lagrangian, which was later on subtracted. That definitively obscures its actual role, which eventually led to some confusion: In [6] situation (ii) was not allowed, whereas the heavy quark hadronic Lagrangian was used for situation (i), which is not correct.

${ }^{9}$ This point was not properly specified in [6].
}

$$
f_{H / 2}^{2}=\frac{\vec{f}_{H}^{2}}{2}+\frac{\mu^{3} N_{c}}{6 \pi^{2}}
$$

where $\bar{f}_{H}^{2}$ is cutoff independent. Notice that in this situation all nonperturbative effects in the small relative momentum region are parametrized by a single nonperturbative constant which is spin and flavor independent. ${ }^{10}$ This is a nontrivial consequence of the $\mathrm{U}\left(4 N_{h f}\right)$ symmetry. The fact that the latter is spontaneously broken down to $\mathrm{U}\left(2 N_{h f}\right) \otimes \mathrm{U}\left(2 N_{h f}\right)$ allows us to know the Green function behavior at low-energy insertion with a single nonperturbative constant since no mass term appears in the pole. All the spin and flavor dependence is explicitly known in the observables.

However, caution must be taken in situation (ii). This is due to the fact that, in this situation, the standard evaluation of nonperturbative contributions in the large relative threemomentum region coming from Eq. (2.15) becomes unreliable. Let us briefly recall the two approximations involved, namely, the multipole expansion and the adiabatic approximation. The first one is an expansion in $\Lambda_{\mathrm{QCD}}$ over the inverse Bohr radius, which has also been assumed to hold throughout. The second one requires the time evolution of the soft gluon fields to be slow in comparison with the energies involved in the Coulomb spectrum. This requirement is in fact the opposite of situation (ii). Thus we are in the unfortunate situation that when we have an excellent parametrization of the nonperturbative effects in the small relative momentum region [Eqs. (5.4) and (5.5)] we lose control of them in the large relative momentum region.

Nevertheless, we envisage a situation where the parametrization (5.4) and (5.5) may be useful. Recall that although the parameter controlling the adiabatic approximation and the parameter controlling the expansion in the hadronic effective Lagrangian are both of order $\Lambda_{\mathrm{QCD}}$, they need not be exactly the same. The former was shown to be $\langle D F D F\rangle /$ $\langle F F\rangle$ in [7] and let $2 \pi \bar{f}_{H}^{2 / 3}$ be the latter. Suppose then that

$$
\begin{gathered}
E_{a b, n}>\left(\frac{\langle D F D F\rangle}{\langle F F\rangle}\right)^{1 / 2}, \\
E_{a b, n}<2 \pi \bar{f}_{H}^{2 / 3} .
\end{gathered}
$$

In such a situation it would be reasonable to use both the adiabatic approximation in the large relative momentum region and the hadronic effective Lagrangian in the small relative momentum region. Some bottomonium, charmonium, and presumably $B_{c}$ states may well be considered in the situation (5.7). However, the mass of the $b$ quark and mainly the mass of the $c$ quark are not large enough to allow for a straightforward application of our formalism to phenomenology. Relativistic and radiative corrections are in general im-

\footnotetext{
${ }^{10}$ Notice also that although at first sight the contributions obtained by substituting Eqs. (5.4) and (5.5) into Eqs. (3.17), (3.27), and (3.33) look like more important than those from the condensate when $m_{Q} \rightarrow \infty$, they are actually not so since the smallness of $\alpha$ required in situation (ii) maintains the condensate contribution dominant. Some statements made in [6] implying the opposite must be corrected.
} 
TABLE I. We display $A_{2}, A_{3}$, and $A_{\mathrm{VL}}$ defined in Eq. (5.10). The last two columns give our results for $m_{b}$ and $a_{b b, 1}^{-1}$.

\begin{tabular}{lccccc}
\hline \hline$\Lambda_{\mathrm{QCD}}^{n f=3}(\mathrm{MeV})$ & $A_{2}(\mathrm{MeV})$ & $A_{3}(\mathrm{MeV})$ & $A_{\mathrm{VL}}(\mathrm{MeV})$ & $m_{b}(\mathrm{MeV})$ & $a_{b b, 1}^{-1}(\mathrm{MeV})$ \\
\hline 200 & -314 & 49 & 25 & 4850 & 1234 \\
250 & -376 & 61 & 18 & 4879 & 1354 \\
300 & -440 & 74 & 13 & 4906 & 1468 \\
\hline \hline
\end{tabular}

portant and this is also so for the nonpertubative corrections due to the gluon condensate [3]. All them must be taken into account.

Let us next discuss the expected size of our contributions. It is not our aim to present a full-fletched phenomenological analysis in order to extract $\bar{f}_{H}^{2}$ from the data, which would definitely be premature as should be clear from the following discussion, but to just give reasonable estimates of the expected magnitude of its contributions. For simplicity, we will concentrate on the mass corrections.

We start with the bottomonium system where our formalism is expected to apply for the lowest-lying states $[3,19]$. We proceed as follows. First of all, we fix $m_{b}$ and $a_{b b, 1}^{-1}$ using the experimental data and the available theoretical results while ignoring the contribution $\delta E_{a b, n}$ in Eq. (3.17). Then we estimate the size of $\delta E_{a b, n}$ by letting $\bar{f}_{H}^{2}$ run within values of the order of $\Lambda_{\mathrm{QCD}}$. We should keep in mind that although we will take $\bar{f}_{H}^{2}$ positive for definiteness, it can also be negative. We extract $m_{b}$ and $a_{b b, 1}^{-1}$ from the selfconsistency equation $a_{b b, 1}\left[\alpha_{s}\left(a_{b b, 1}^{-1}\right)\right]=a_{b b, 1}$ and the $Y(1 s)$ mass. We use the following equation to fit the latter:

$$
m_{\Upsilon(1 S)}=2 m_{b}+A_{2}+A_{3}+A_{\mathrm{VL}} .
$$

where

$$
\begin{gathered}
a_{b b, 1}^{-1}=\frac{m_{b} C_{f} \widetilde{\alpha}\left(a_{b b, 1}^{-1}\right)}{2}, \\
A_{2}=-2 m_{b} \frac{C_{f}^{2} \widetilde{\alpha}^{2}\left(a_{b b, 1}^{-1}\right)}{8}, \\
A_{3}=-2 m_{b} \frac{C_{f}^{2} \beta_{0} \alpha^{2}\left(a_{b b, 1}^{-1}\right) \widetilde{\alpha}\left(a_{b b, 1}^{-1}\right)}{8 \pi} \\
\times\left(\ln \left[\frac{\left(a_{b b, 1}^{-1}\right)}{m_{b} C_{f} \widetilde{\alpha}\left(a_{b b, 1}^{-1}\right)}\right]+1-\gamma_{E}\right), \\
A_{\mathrm{VL}}=m_{b} \frac{e_{10} \pi\left\langle\alpha_{s} G^{2}\right\rangle}{\left[m_{b} C_{f} \widetilde{\alpha}\left(a_{b b, 1}^{-1}\right)\right]^{4}} .
\end{gathered}
$$

We have taken the formulas above, ${ }^{11}$ which include relativistic, radiative, and the VL nonperturvative corrections, from [3]. We allow for different values of $\Lambda_{\mathrm{QCD}}$ and give the relative weight of each contribution in Table I.

\footnotetext{
${ }^{11}$ However, we have not taken into account the contributions of order $O\left(\alpha^{4}, \alpha^{5}\right)$ given in [3] since the complete calculation at this order is still lacking.
}

Let us next assume that we are in the situation (5.7). As mentioned before, this may well be the case for the $Y(1 S)$, $Y(2 S), \chi_{b}(1 P), J / \psi$ (and $\left.\eta_{c}\right)$, and $B_{c}$ (and $B_{c}^{*}$ ). If we let $\bar{f}_{H}^{2 / 3}$ run between the values

$$
E_{a b, n}<2 \pi \bar{f}_{H}^{2 / 3}<a_{a b, n}^{-1},
$$

we can give an estimate of $\delta E_{a b, n}$. If we allow $\bar{f}_{H}^{2 / 3}$ between 100 and $150 \mathrm{MeV}$, our results turn out to be quite stable under values of $\Lambda_{\mathrm{QCD}}^{n f=3}$ between 200 and $300 \mathrm{MeV}$. We obtain

$$
-9 \mathrm{MeV}<\delta E_{b b, 1}<-2 \mathrm{MeV}
$$

where the explicit expresion used for calculating $\delta E_{a b, n}$ reads

$$
\delta E_{a b, n}=-4 \mu_{a b} \frac{16 \pi \bar{f}_{H}^{2}}{N_{c} C_{f} \widetilde{\alpha}_{s}\left(a_{a b, n}^{-1}\right)}\left(\frac{n}{2 \mu_{a b}}\right)^{3} .
$$

Although the smallness of the result above is discouraging at first sight, it justifies the procedure used and makes it selfconsistent.

For $n=2$ we obtain

$$
-55 \mathrm{MeV}<\delta E_{b b, 2}<-15 \mathrm{MeV} \text {. }
$$

Recall that only the $s$-wave states receive this correction. If the sign of $\bar{f}_{H}^{2}$ was negative, the signs above would be reversed. This would help to understand the mass difference between the $\chi_{b}(1 P)$ and the $Y(2 S)$.

Let us finally give some estimates for $\delta E_{c c, 1}$ and $\delta E_{b c, 1}$ corresponding to the $J / \Psi$ (and $\eta_{c}$ ) and the $B_{c}$ (and $B_{c}^{*}$ ) ground states. We have taken the mass of the charm quark, $m_{c}=1570 \mathrm{MeV}$, as given in Ref. [3]. For the $J / \Psi$ we find

$$
\delta E_{c c, 1} \sim-42 \mathrm{MeV},
$$

taking $\Lambda_{\mathrm{QCD}}^{n f=3}=300 \mathrm{MeV}, a_{c c, 1}^{-1}=848 \mathrm{MeV}$, and $\bar{f}_{H}^{2 / 3}=150$ $\mathrm{MeV}$. For the $B_{c}$ we find

$$
\delta E_{b c, 1} \sim-23 \mathrm{MeV}
$$

taking $\Lambda_{\mathrm{QCD}}^{n f=3}=300 \mathrm{MeV}, a_{b c, 1}^{-1}=1013 \mathrm{MeV}$, and $\bar{f}_{H}^{2 / 3}=150$ $\mathrm{MeV}$.

The above contributions for the energy shifts are, on the one hand, small enough to make us confident that our results are under control and, on the other hand, large enough to hope for its eventual observation. However, it is important to realize that the $\mathrm{VL}$ contributions are exceedingly large for $Y(2 S), \chi_{b}(1 P), J / \psi\left(\right.$ and $\left.\eta_{c}\right)$, and $B_{c}$ (and $\left.B_{c}^{*}\right)$. We suspect that the framework used so far to calculate the VL contributions in the large relative momentum region is not appropiated for these states. We believe that in order to make real- 
istic QCD-based predictions for these states one should devise a reliable approximation in the large relative momentum region to deal with situation (ii) above, namely, inverse Born radius and energy larger and smaller than $\Lambda_{\mathrm{QCD}}$, respectively. Work in this direction is in progress [20].

\section{CONCLUSIONS}

We are confident that the theoretical framework above is going to be useful for an eventual QCD-based formalism attempting to encompass situations where the Coulomb energy is large (small $n$ ) and situations where it is small (large $n$ ) with respect to $\Lambda_{\mathrm{QCD}}$ in heavy quarkonium. Even more, this formalism could also be useful in order to obtain explicitly the perturbative Coulomb corrections to the nonperturbative heavy quarks bound states (large $n$ ).

Our formalism is clearly inspired by the Wilson renormalization group approach. We separate the fields into large and small momentum components by an explicit cutoff and work out what the effective action for the latter is. However, there is an important point which makes our formalism rather peculiar: Integrating out the large momentum components does not give rise to local counterterms only. There is nontrivial physics in the ultraviolet, namely, Coulomb-type bound states. As far as we know, this is the first example of a Wilsonian approach where effects due to bound states have been taken into account.

Let us finally summarize the main contributions of this paper. Elaborating on the ideas first presented in [6], we have produced a detailed derivation of the effective theory governing the small relative momentum degrees of freedom in heavy quarkonium. In particular this includes an interaction term, which had been overlooked before, that leads to a few corrections in the observables. We have proven the cutoff independence of the formalism. We have also discussed in detail when nonperturbative contributions which cannot be expressed in terms of local condensates arise, namely, when a description in terms of a heavy quark hadronic theory is adequate. Our preliminary estimations suggests that these contributions lead to energy shifts of a few tens of $\mathrm{MeV}$. Unfortunately, more theoretical work is necessary to establish them from the data. This is mainly due to the lack of control on the nonperturbative effects in the large relative momentum region of most of the systems where our approach should apply, namely, $\Upsilon(2 S), \chi_{b}(1 P), J / \psi$ (and $\eta_{c}$ ), and $B_{c}$ (and $B_{c}^{*}$ ). Work in this direction is in progress [20].

\section{ACKNOWLEDGMENTS}

J.S. thanks Professor G. Veneziano and the CERN theory group for their hospitality while this work was written up. A.P. acknowledges a financial support from CIRIT. Financial support from CICYT, Contract No. AEN95-0590, and financial support from CIRIT, Contract No. GRQ93-1047, is acknowledged.
[1] C. T. H. Davies, K. Hornbostel, A. Langau, G. P. Lepage, A. Liedsey, C. J. Morningstar, J. Shigemitsu, and J. Sloan, Phys. Rev. Lett. 73, 2654 (1994); C. T. H. Davies, K. Hornbostel, A. Langau, G. P. Lepage, A. Liedsey, J. Shigemitsu, and J. Sloan, Phys. Rev. D 50, 6963 (1994); C. T. H. Davies, A. Liedsey, P. McCallum, K. Hornbostel, G. P. Lepage, J. Shigemitsu, and J. Sloan, in Lattice 95, Proceedings of the International Symposium, Melbourne, Australia, 1995, edited by T. D. Kieu et al. [Nucl. Phys. B Proc. Suppl. 47 (1996)].

[2] M. B. Voloshin, Int. J. Mod. Phys. A 10, 2865 (1995).

[3] S. Titard and F. J. Ynduráin, Phys. Rev. D 49, 6007 (1994); 51, 6348 (1995); Phys. Lett. B 351, 541 (1995); Y. A. Simonov, S. Titard, and F. J. Yndurain, ibid. 354, 435 (1995).

[4] C. A. A. Nunes, F. S. Navarra, P. Ring, and M. Schaden, "Nonperturbative effects in heavy quarkonia," report, hep-ph 9409389 (unpublished), and references therein.

[5] S. Narison, "Heavy quarkonia mass-splittings in QCD: gluon condensate, $\alpha_{s}$ and 1/m-expansion," Report No. PM 95/51, hep-ph 9512348 (unpublished).

[6] A. Pineda and J. Soto, Phys. Rev. D 53, 3983 (1996).

[7] M. B. Voloshin, Nucl. Phys. B154, 365 (1979); Sov. J. Nucl. Phys. 36, 143 (1982).

[8] H. Leutwyler, Phys. Lett. 98B, 447 (1981).

[9] G. Curci, A. Di Giacomo, and G. Paffuti, Z. Phys. C 18, 135 (1983); M. Schiestl and H. G. Dosch, Phys. Lett. B 209, 85 (1988); J. Liu, H. Huang, and R. Wang, Phys. Rev. D 49, 3474 (1994).
[10] I. I. Bigi, M. A. Shifman, N. G. Uraltsev, and A. I. Vainshtein, Phys. Rev. D 50, 2234 (1994); M. Shifman, in Continuous Advances in $Q C D$, edited by A. V. Smilga (World Scientific, Singapore, 1994).

[11] G. Burdman and J. F. Donoghue, Phys. Lett. B 280, 287 (1992); Tung-Mow Yan et al., Phys. Rev. D 46, 1148 (1992); 47, 145 (1992); Peter Cho, Phys. Lett. B 285, 145 (1992).

[12] J. Soto and R. Tzani, Int. J. Mod. Phys. A 9, 4949 (1994).

[13] M. B. Voloshin and M. A. Shifman, Yad. Fiz. 45, 463 (1987) [Sov. J. Nucl. Phys. 45, 292 (1987)]; H. D. Politzer and M. B. Wise, Phys. Lett. B 206, 681 (1988); 208, 504 (1988); N. Isgur and M. B. Wise, ibid. 232, 113 (1989); 237, 527 (1990); E. Eichten and B. Hill, ibid. 234, 511 (1990); H. Georgi, ibid. 240, 447 (1990); B. Grinstein, Nucl. Phys. B339, 253 (1990).

[14] B. Grinstein, in High Energy Phenomenology, Proceedings of the Workshop, Mexico City, Mexico, 1991, edited by M. A. Perez et al. (World Scientific, Singapore, 1992), p. 161; in Intersections Between Particle and Nuclear Physics, Proceedings of the International Conference, Tucson, Arizona, 1991, edited by W. T. H. van Oers (AIP, New York, 1996), p. 112; T. Mannel, Chin. J. Phys. 31, 1 (1993); M. Neubert, Phys. Rep. 245, 259 (1994).

[15] A. Pineda and J. Soto, Phys. Lett. B 361, 95 (1995).

[16] W. E. Caswell and G. P. Lepage, Phys. Lett. B 167, 437 (1986); G. P. Lepage and B. A. Thacker, in Field Theory on the Lattice, Proceedings of the International Symposium, Seil- 
loc, France, 1987, edited by A. B. Billoire et al. [Nucl. Phys. Proc. Suppl. 4, 199 (1988)]; G. T. Bodwin, E. Braaten, and G. P. Lepage, Phys. Rev. D 51, 1125 (1995).

[17] J. J. Sakurai, Modern Quantum Mechanics (Addison-Wesley, New York, 1985).
[18] H. A. Bethe and E. E. Salpeter, Quantum Mechanics of One and Two-Electron Atoms (Springer-Verlag, Berlin, 1957).

[19] J. Pantaleone, S.-H. H. Tye, and Y. J. Ng, Phys. Rev. D 33, 777 (1986).

[20] A. Pineda and J. Soto (in preparation). 